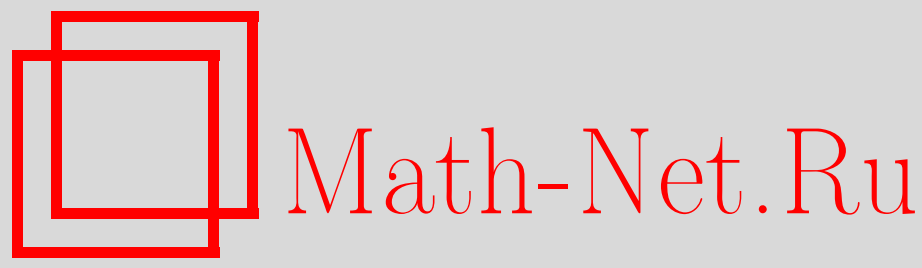

М. Е. Шапошников, Асимптотическая безопасность гравитации и масса бозона Хиггса, ТМФ, 2012, том 170, номep 2, 280-291

DOI: https://doi.org/10.4213/tmf6766

Использование Общероссийского математического портала Math-Net.Ru подразумевает, что вы прочитали и согласны с пользовательским соглашением http://www.mathnet.ru/rus/agreement

Параметры загрузки:

IP : 54.237 .59 .107

26 апреля 2023 г., 15:52:05

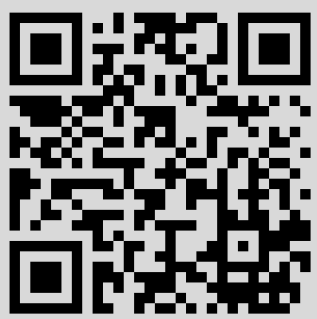




\title{
АСИМПТОТИЧЕСКАЯ БЕЗОПАСНОСТЬ ГРАВИТАЦИИ И МАССА БОЗОНА ХИГГСА
}

\begin{abstract}
Если гравитация является асимптотически безопасной теорией, то окончательной теорией может оказаться просто Стандартная модель (минимально дополненная несколькими легкими частицами с целью согласовать массы и осцилляции нейтрино, темное вещество и барионную асимметрию Вселенной) плюс гравитация. Если это действительно так, то массу бозона Хиггса можно предсказать, $m_{\mathrm{H}}=m_{\min } \simeq 130$ ГэВ с точностью всего до нескольких ГэВ, или ограничить интервалом $m_{\min }<m_{\mathrm{H}}<m_{\max } \simeq 174$ ГэВ.
\end{abstract}

Ключевые слова: асимптотическая безопасность, бозон Хиггса.

\section{1. ВВЕДЕНИЕ}

Наиболее минималистский подход к квантовой гравитации связан с понятием асимптотической безопасности [1]. Хотя общая теория относительности не может быть ренормирована методами теории возмущений, она может существовать как непертурбативная теория поля, имея нетривиальную ультрафиолетовую фиксированную точку (см. обзорную работу [2]). В рамках этого предположения есть возможность эффективно описать все взаимодействия в природе. Можно предположить, что новая физика отсутствует на промежуточных масштабах энергии между масштабами Ферми и Планка $M_{\mathrm{P}}=2.44 \cdot 10^{18}$ ГэВ. Все подтвержденные наблюдаемые сигналы, свидетельствующие в пользу существования физики, уходящей за пределы Стандартной модели (CM) (такие, как массы и осцилляции нейтрино, темная материя и темная энергия, барионная асимметрия Вселенной и инфляция), можно отнести к новой физике, существующей ниже масштаба электрослабых взаимодействий (см. обзоры [3], [4] и имеющиеся в них ссылки). Минимальная модель $\nu \mathrm{MCM}$ - содержит наряду с частицами СМ три сравнительно легких синглетных фермиона Майорана и дилатон. Эти фермионы могли бы отвечать за массы нейтрино, темное вещество и барионную асимметрию Вселенной. Дилатон может привести к динамической темной энергии [5], [6] и реализует спонтанно нарушеннную масштабную инвариантность, которая либо возникает при использовании космологического подхода к фиксированной точке [5], [7], либо является точной квантовой

*École Polytechnique Fédérale de Lausanne, Lausanne, Switzerland.

E-mail: Mikhail.Shaposhnikov@epfl.ch 
симметрией [8], [9]. Инфляция может иметь место или из-за присутствия частицы Хиггса в CM [10], или вследствие асимптотически безопасного характера гравитации [11]. Еще одну часть новой физики, включая, например, сильную СР-проблему или проблему аромата, можно связать с масштабом Планка.

Однако такая точка зрения сталкивается с препятствием, связанным с проблемой полюсов Ландау для некоторых взаимодействий в СМ (или в $\nu \mathrm{MCM}$ ). А именно, константы калибровочного взаимодействия группы $U(1) g^{\prime} \equiv g_{1}$, самодействия Хиггса $\lambda$, а также взаимодействия Юкавы $y_{\mathrm{t}}$ (это взаимодействие наиболее ощутимо для топ-кварка) не являются асимптотически свободными. Этот факт делает невозможной формулировку фундаментальной СМ, оставляя ей роль эффективной теории поля, справедливой только ниже некоторого энергетического масштаба.

В настоящей работе, основанной на статье [12], мы обсуждаем сценарий, который может преодолеть указанную трудность и позволяет предсказать величину массы бозона Хиггса, которую можно проверить на Большом адронном коллайдере (БАК). В разделе 2 дан краткий обзор асимптотической безопасности теории; в разделе 3 мы обсуждаем, каким образом асимптотическая безопасность СМ может возникнуть благодаря комбинации СМ с асимптотически безопасной гравитацией и предсказываем массу бозона Хиггса; выводы приведены в разделе 4.

\section{2. АСИМПТОТИЧЕСКАЯ БЕЗОПАСНОСТЬ}

Поиск "хорошей" квантовой теории поля можно вести следующим образом.

1. Взять некоторый конкретный набор квантовых полей и написать наиболее общий лагранжиан, учитывающий выбранные симметрии и включающий операторы произвольной размерности.

2. Вычислить все амплитуды рассеяния во всех порядках теории возмущений.

3. Потребовать, чтобы эта теория была унитарно- и лоренц-инвариантна, а также удовлетворяла принципу причинности. Это приведет к бесконечному числу условий, налагаемых на бесконечное число констант связи, которые определяют теорию.

4. Решить эти уравнения совместности. Следует надеяться, что теория будет характеризоваться конечным числом существенных параметров - констант связи, что даст возможность делать предсказания.

Конечно, реализовать эту программу очень сложно, если вообще возможно. Один из подходов основан на ренормализационной группе (РГ) [1]. Введем безразмерные константы связи $g_{i}$ для всех членов в действии: $g_{i}=\mu^{D_{i}} G_{i}$, где $D_{i}$ - каноническая размерность константы связи $G_{i}$, а $\mu$ - произвольный параметр, имеющий размерность массы. Уравнения РГ выводятся из требования, чтобы физические амплитуды не зависели от $\mu$. Это приводит к бегу констант связи $g=\left\{g_{i}\right\}$ в соответствии с уравнениями

$$
\mu \frac{\partial g_{i}}{\partial \mu}=\beta_{i}(g)
$$

и фиксирует $\beta$-функции.

Ренормируемые асимптотически свободные теории соответствуют гауссовым ультрафиолетовым фиксированным точкам: существенные константы связи $g_{i}(\mu) \rightarrow 0$ при $\mu \rightarrow \infty$. Число таких констант связи конечно - допустимы только операторы размерности не выше 4 . К хорошо известным примерам асимптотически свободных теорий относятся КХД, некоторые теории великого объединения и ренормируемые теории в пространстве-времени размерностей 2 и 3. 
Асимптотически безопасные теории связаны с негауссовыми ультрафиолетовыми фиксированными точками $g^{*} \neq 0: \beta_{i}\left(g^{*}\right)=0$. Хотя эти теории не перенормируемы, они дают возможность делать предсказания в том случае, когда размерность критической поверхности в пространстве констант связи (точки, которые притягиваются к $g^{*}$ при $\left.\mu \rightarrow \infty\right)$ конечна. Известными примерами являются скалярная теория поля в трех измерениях с фиксированной точкой Вильсона-Фишера (размерность критической поверхности равна 2), нелинейная сигма-модель [13] и гравитация в $2+\epsilon$ измерениях [1], [14]-[16].

Сложно определить, является ли некоторая теория асимптотически безопасной, поскольку стандартное разложение теории возмущений не работает. К стандартным методам относятся $\epsilon$-разложение [17], численные расчеты на решетке [18], [19] и метод функциональной РГ [20], [21]. Оригинальная гипотеза Вайнберга об асимптотической безопасности гравитации основана на $\epsilon$-разложении. Начатые в работе [22] интенсивные исследования функциональной РГ для гравитации были продолжены в работах [23], [24], где приведены свидетельства в ее пользу. В дальнейшем мы будем считать, что гравитация действительно является асимптотически безопасной.

\section{3. АСИМПТОТИЧЕСКИ БЕЗОПАСНАЯ СТАНДАРТНАЯ МОДЕЛЬ И МАССА БОЗОНА ХИГГСА}

СМ не является ни асимптотически свободной, ни асимптотически безопасной. В ней присутствуют полюсы Ландау константы связи калибровочной группы $U(1)$, констант взаимодействия Юкавы и самодействия Хиггса. Однако не исключено, что комбинация СМ с асимптотически безопасной гравитацией может изменить ситуацию и привести к непротиворечивой теории. Далее мы обсудим, как это может произойти. Мы сосредоточимся на эволюции калибровочных констант связи СМ $g_{1}$, $g \equiv g_{2}$ и $g_{3}$, относящихся к группам $U(1), S U(2)$ и $S U(3)$ соответственно, а также на константе связи $\lambda$ самодействия Хиггса и константе связи $y_{\mathrm{t}}$ взаимодействия Юкавы для топ-кварков. Калибровочные константы связи мы фиксируем в соответствии с их экспериментальными значениями при малых энергиях, а $\lambda$ и $y_{\mathrm{t}}$ пока оставим не определенными.

В уравнения РГ для самодействующей материи входит вклад от гравитационного сектора [1], [25], [26]. В общем случае уравнения РГ для указанных констант связи с учетом гравитационных поправок имеют вид

$$
\frac{d h}{d t}=\beta_{h}^{\mathrm{SM}}+\beta_{h}^{\mathrm{grav}}
$$

где $t=\ln \mu, h$ - любая из констант связи $g_{i}, \lambda$ или $y_{\mathrm{t}}, \beta_{h}^{\mathrm{SM}}-$ вклад СМ и $\beta_{h}^{\text {grav }}-$ гравитационные поправки. В однопетлевом приближении

$$
\begin{aligned}
& \beta_{1}^{\mathrm{SM}}=\frac{1}{16 \pi^{2}} \frac{41}{6} g_{1}^{3}, \quad \beta_{2}^{\mathrm{SM}}=-\frac{1}{16 \pi^{2}} \frac{19}{6} g_{2}^{3}, \quad \beta_{3}^{\mathrm{SM}}=-\frac{1}{16 \pi^{2}} 7 g_{3}^{3}, \\
& \beta_{y}^{\mathrm{SM}}=\frac{1}{16 \pi^{2}}\left[\frac{9}{2} y_{\mathrm{t}}^{3}-8 g_{3}^{2} y_{\mathrm{t}}-\frac{9}{4} g_{2}^{2} y_{\mathrm{t}}-\frac{17}{12} g_{1}^{2} y_{\mathrm{t}}\right], \\
& \beta_{\lambda}^{\mathrm{SM}}=\frac{1}{16 \pi^{2}}\left[24 \lambda^{2}+12 \lambda y_{\mathrm{t}}^{2}-9 \lambda\left(g_{2}^{2}+\frac{1}{3} g_{1}^{2}\right)-6 y_{\mathrm{t}}^{4}+\frac{9}{8} g_{2}^{4}+\frac{3}{8} g_{1}^{4}+\frac{3}{4} g_{2}^{2} g_{1}^{2}\right] .
\end{aligned}
$$


Структуру гравитационных поправок можно извлечь из размерностного анализа:

$$
\beta_{h}^{\text {grav }}=\frac{a_{h}}{8 \pi} \frac{\mu^{2}}{M_{\mathrm{P}}^{2}(\mu)} h,
$$

где $a_{1}, a_{2}, a_{3}, a_{y}$ и $a_{\lambda}$ - некоторые константы (аномальные размерности), соответствующие $g_{1}, g_{2}, g_{3}, y_{\mathrm{t}}$ и $\lambda$, а $M_{\mathrm{P}}^{2}(\mu)$ - бегущая масса Планка. Исследования функциональной РГ приводят к выводу о следующей характерной зависимости гравитационной постоянной или массы Планка от масштаба:

$$
M_{\mathrm{P}}^{2}(\mu)=M_{\mathrm{P}}^{2}+2 \xi_{0} \mu^{2},
$$

где $\xi_{0}$ - число, точное значение которого для нас не существенно. Путем численного решения уравнений функциональной РГ авторы работ [22], [25], [27] нашли, что $\xi_{0} \approx 0.024$. Итак, в случае большого переданного импульса $q^{2} \gg M_{\mathrm{P}}^{2}$ эффективная гравитационная константа $G_{N}\left(q^{2}\right)$ масштабируется как $1 / 16 \pi \xi_{0} q^{2}$, обеспечивая регулярное поведение высокоэнергетических амплитуд рассеяния. Расчеты различных аномальных размерностей проводились в статьях [25]-[32]. Отметим, однако, что среди авторов нет единого мнения о величине и даже о знаке коэффициентов $a_{i}$. Более того, определения констант взаимодействий материи не одни и те же в разных статьях. Коэффициенты $a_{i}$, найденные в различных работах, зависят от используемой калибровки и от формы обрезания функциональных уравнений РГ. Мы предполагаем, что со временем появится возможность дать калибровочно-инвариантные определения этих констант взаимодействий. Наиболее вероятно, что они будут основаны на калибровочно-инвариантных высокоэнергетических амплитудах рассеяния, как предлагается в работе [1]. Подчеркнем, что такое определение взаимодействий не совпадает с определением, основанным на схеме минимальных вычитаний (см. статью [33]).

Бег различных констант взаимодействий в СМ можно разделить на два режима. До масштаба $\mu^{2} \sim M_{\mathrm{P}}^{2}$ гравитационные поправки к бета-функциям в СМ подавляются множителем $\mu^{2} / M_{\mathrm{P}}^{2}$ и поэтому являются малыми. Константы взаимодействий изменяются логарифмически до масштаба $\mu^{2} \sim M_{\mathrm{P}}^{2}$. Для $\mu^{2} \gtrsim M_{\mathrm{P}}^{2}$ гравитационные поправки становятся важными. Если преобладает гравитационная часть бета-функций и $\mu^{2} \gtrsim M_{\mathrm{P}}^{2} / 2 \xi_{0}$, то изменение происходит по степенному закону

$$
h \propto \mu^{a_{h} / 16 \pi \xi_{0}} .
$$

Ясно, что знаки аномальных размерностей $a_{h}$ играют решающую роль для справедливости СМ при любых масштабах энергии.

Сначала рассмотрим калибровочный сектор. Для простоты возьмем $a_{1}=a_{2}=$ $a_{3}=a$, что возникает в однопетлевых расчетах в силу универсальности гравитационных взаимодействий. Тогда все калибровочные константы связи становятся асимптотически свободными при условии, что $a<a^{\text {crit }}, a^{\text {crit }} \simeq-0.013$ (величина $a^{\text {crit }}$ соответствует фиксированной точке $g_{1}^{*} \simeq 0.5$ для бега однопетлевой константы связи группы $U(1)$, если он начинается с экспериментального значения, полученного при низких энергиях). Если это так, то проблема полюсов Ландау для константы связи группы $U(1)$ решается с помощью гравитационного вклада в поток РГ. В самом 
деле, расчеты, проведенные в работах [28], [26], дают отрицательный знак для $a$, причем $|a| \sim 1$. Далее мы предположим, что

$$
a<a^{\text {crit }} \text {. }
$$

В этом случае значения калибровочных констант связи предсказать нельзя. Если $a=a^{\text {crit }}$, то величина константы связи группы $U(1)$ предсказуема.

Рассмотрим теперь взаимодействие Юкавы для топ-кварков. Положив, к примеру, $a=-1$ и взяв (среднее) экспериментальное значение массы топ-кварка $m_{\mathrm{t}}=$

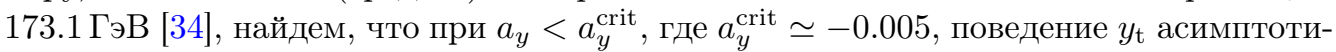
чески свободно, при $a_{y}=a_{y}^{\text {crit }}$ оно соответствует негауссовой фиксированной точке с $y_{\mathrm{t}}^{*} \simeq 0.38$, а при $a_{y}>a_{y}^{\text {crit }}$ поведение определяется полюсом Ландау. Критическое значение величины $a_{y}$ лишь слабо чувствительно к $a$. Например, при $a=-0.02$ получим $a_{y}^{\text {crit }} \simeq-0.002$ и $y_{\mathrm{t}}^{*} \simeq 0.25$. При уменьшении массы топ-кварка соответствующее значение $a_{y}^{\text {crit }}$ еще больше приближается к нулю, а при увеличении $m_{\mathrm{t}}$ параметр $a_{y}^{\text {crit }}$ отодвигается от нуля.

Предположим, что $a_{y}>a_{y}^{\text {crit }}$. Тогда, чтобы обеспечить непротиворечивость теории на всех энергетических масштабах, следует положить $y_{\mathrm{t}}=0$. Это соответствует безмассовому топ-кварку, что расходится с данными эксперимента. Другими словами, если это происходит, то нужно отбросить предположение об отсутствии новой физики между масштабами Ферми и Планка и модифицировать ту часть потока РГ, которая соответствует $y_{\mathrm{t}}$. Таким образом, гипотеза о фундаментальном характере $\mathrm{CM}$ или $\nu \mathrm{MCM}$ может быть верной только в случае $a_{y} \leqslant a_{y}^{\text {crit }} . \mathrm{K}$ сожалению, мы были не в состоянии извлечь надежное значение и знак величины $a_{y}$ из существующей литературы. Например, в работе [35] показано, что гравитационные вклады делают взаимодействие Юкавы асимптотически свободным в квантовой $R^{2}$-гравитации с материей. В статье [32] рассмотрена эволюция константы связи Юкавы под воздействием гравитации в подходе функциональной РГ с использованием гравитационного действия типа Эйнштейна-Гильберта и получено, что параметр $a_{y}$ в различных калибровках имеет разные знаки. В силу этих неопределенностей в дальнейшем мы просто предположим, что $a_{y}<a_{y}^{\text {crit }}$. Как и в случае константы связи для группы $U(1)$, особый случай $a_{y}=a_{y}^{\text {crit }}$ позволил бы предсказать значение $m_{\mathrm{t}}$.

Обратимся к поведению константы скалярного самодействия $\lambda$. Гравитационные поправки могут способствовать выдвижению CM на статус фундаментальной теории, если только изменение $\lambda$ не приводит к каким-нибудь патологиям вплоть до масштаба Планка. Другими словами, полюсы Ландау должны отсутствовать для $k \lesssim M_{\mathrm{P}}[36]-[39]$, а константа $\lambda$ должна быть положительной для всех импульсов вплоть до $M_{\mathrm{P}}$ [40]-[42], обеспечивая стабильность электрослабого вакуума. Существует обширная область параметров на плоскости $\left(m_{\mathrm{H}}, m_{\mathrm{t}}\right)$, где оба условия удовлетворены. Вблизи экспериментального значения массы топ-кварка она описывается условием

$$
m_{\min }<m_{\mathrm{H}}<m_{\max }
$$

Здесь

$$
\begin{aligned}
& m_{\min }=129.5+\frac{m_{\mathrm{t}}-173.1}{2.1} \cdot 4.1-\frac{\alpha_{\mathrm{s}}-0.1183}{0.002} \cdot 1.5, \\
& m_{\max }=174.0+\frac{m_{\mathrm{t}}-173.1}{2.1} \cdot 0.6-\frac{\alpha_{\mathrm{s}}-0.1183}{0.002} \cdot 0.1
\end{aligned}
$$




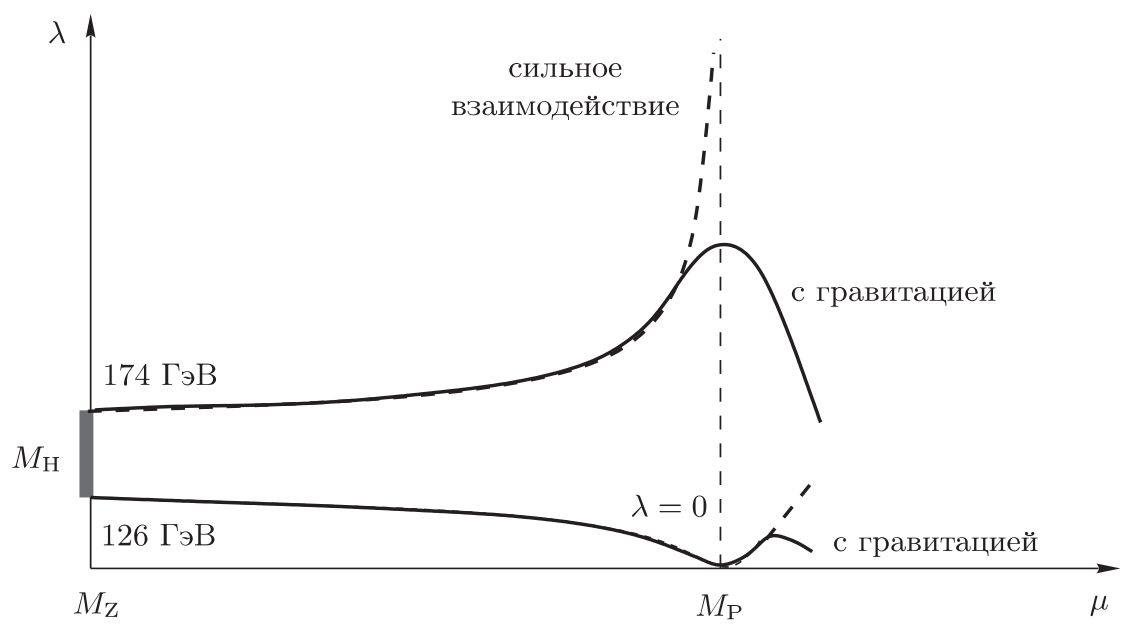

Рис. 1. Эволюция константы самодействия Хиггса $\lambda$ в СM, дополненной асимптотически безопасной гравитацией, для случая отрицательной аномальной размерности $a_{\lambda}$.

(в ГэВ) и $\alpha_{\mathrm{s}}$ - константа сильного взаимодействия на масштабе массы $Z$-бозона; теоретическая неопределенность для $m_{\min }$ равна \pm 2.2 ГэВ. Эти данные взяты из результатов недавно проведенного двухпетлевого анализа [43] (см. также [44], [45] и более ранние вычисления в работах [46]-[49]). Величина $m_{\max }$ соответствует (в некоторой степени произвольному) критерию $\lambda\left(M_{\mathrm{P}}\right)<6$. Допустимая область содержит также очень малые массы топ-кварка и бозона Хиггса, которые исключаются в соответствии с экспериментальными данными.

Предположим сначала, что константа $a_{\lambda}$ отрицательна и достаточно велика по абсолютной величине:

$$
a_{\lambda}<a_{\lambda}^{\text {crit }} \simeq-\frac{24 \xi_{0} \lambda\left(M_{\mathrm{P}}\right)}{\pi}
$$

(величина $a_{\lambda}^{\text {crit }} \simeq-1$, если $\lambda\left(M_{\mathrm{P}}\right) \simeq 6$ ). Тогда взаимодействие Хиггса асимптотически свободно во всей области пространства параметров, ограниченной условием (5). Гравитационный вклад устраняет поведение, обусловленное полюсами Ландау при энергиях, превышающих массу Планка (см. рис. 1). Для $m_{\mathrm{t}}=173.1$ ГэВ (напомним, что масса топ-кварка не может быть предсказана, если $\left.a_{y}<a_{y}^{\text {crit }}\right)$, пренебрегая погрешностью в теоретических расчетах и в расчетах $\alpha_{\mathrm{s}}$, получим, что масса бозона Хиггса должна лежать в интервале [129.5, 174.0] ГэВ. Если фактическая величина параметра $a_{\lambda}^{\text {crit }}$ меньше единицы, то верхний предел для массы бозона Хиггса понижается.

Наиболее интересная ситуация реализуется при положительных значениях константы $a_{\lambda}$, что приводит к конкретному предсказанию масс бозона Хиггса и топкварка. В пользу этого свидетельствуют расчеты, проведенные в работах [25], [27]. Согласно этим расчетам $a_{\lambda} \simeq 3.1$. Вклад с тем же знаком и похожей величиной был найден ранее в работе [50].

Объясним структуру решения уравнения РГ для $\lambda$ в этом случае. Из-за положительности $a_{\lambda}$ общее решение уравнения (1) расходится при $\mu \rightarrow \infty$, что ведет к 
противоречиям в теории. Однако может существовать частное решение, приводящее к тому, что $\lambda \rightarrow 0$ (или, в особом случае, $\lambda \rightarrow$ const $\neq 0$ ) в ультрафиолетовом пределе. Легко видеть, что требуемое поведение возможно, только если вклад от топ-кварка, появляющийся вместе с отрицательным знаком $\beta_{\lambda}^{\mathrm{SM}}$, преобладает над калибровочным вкладом при $t \rightarrow \infty$, приводя к ограничению

$$
a \leqslant a_{y} \leqslant a_{y}^{\text {crit }}
$$

Если $a<a_{y}<a_{y}^{\text {crit }}$, то ультрафиолетовая асимптотика для $\lambda$ выглядит так:

$$
\lambda(\mu) \approx \frac{6 y_{\mathrm{t}}^{4}(\mu) \xi_{0}}{\pi a_{\lambda}}
$$

При $a_{y}=a$ имеем $\lambda(\mu) \propto \mu^{a / 2 \pi \xi_{0}}$, тогда, как и при $a_{y}=a_{y}^{\text {crit }}$, существует негауссова фиксированная точка $\lambda^{*}$, которая удовлетворяет уравнению

$$
24 \lambda^{* 2}+12 \lambda^{*} y_{\mathrm{t}}^{* 2}-6 y_{\mathrm{t}}^{* 4}+\frac{\pi a_{\lambda} \lambda^{*}}{\xi_{0}}=0 .
$$

Если $a_{\lambda} \geqslant 0$, то амплитуда скалярного самодействия не превышает $\lambda^{*}<0.3 y_{\mathrm{t}}^{* 2}$.

Подведем итог: для любого заданного набора аномальных размерностей $a_{h}$, удовлетворяющего условиям (4), (8), и любого $a_{\lambda} \geqslant 0$ существует единственное значение константы связи низкоэнергетического скалярного самодействия, которое приводит к непротиворечивой теории. Это означает, что можно предсказать массу бозона Хиггса. Чтобы найти $m_{\mathrm{H}}$, следует решить уравнения РГ, фиксируя начальные значения (скажем, при $\mu$, равном массе $M_{\mathrm{Z}} Z$-бозона) для констант связи калибровочных взаимодействий и взаимодействия Юкавы и подобрать $\lambda$ так, чтобы она обращалась в нуль при $\mu \rightarrow \infty$ или приближалась к фиксированной точке $\lambda^{*}$. Кроме того, приемлемы только траектории РГ с положительными значениями $\lambda$.

Следующее рассуждение позволяет локализовать значение массы бозона Хиггса и приводит к непротиворечивой теории. Уравнение РГ для $\lambda$, удовлетворяющее требованию асимптотической безопасности, можно написать в виде интегрального уравнения

$$
\lambda(\mu)=-\int_{\mu}^{\infty} \frac{d \mu^{\prime}}{\mu^{\prime}}\left(\frac{1+2 \xi_{0} \mu^{2} / M_{\mathrm{P}}^{2}}{1+2 \xi_{0}\left(\mu^{\prime}\right)^{2} / M_{\mathrm{P}}^{2}}\right)^{a_{\lambda} / 32 \pi \xi_{0}} \beta_{\lambda}^{\mathrm{SM}}\left(h\left(\mu^{\prime}\right)\right) .
$$

Предполагая, что все взаимодействия стремятся к нулю по закону (3), мы приходим к следующему граничному условию при $\mu=M_{\mathrm{P}}$ :

$$
\lambda\left(M_{\mathrm{P}}\right)=-C \beta_{\lambda}^{\mathrm{SM}}\left(h\left(M_{\mathrm{P}}\right)\right),
$$

где $C$ - положительная константа порядка 1 . Поскольку $\beta_{\lambda}^{\mathrm{SM}} \ll \lambda$ в точке $k=M_{\mathrm{P}}$, это условие можно заменить следующим:

$$
\lambda\left(M_{\mathrm{P}}\right) \approx 0
$$

Итак, бег константы связи $\lambda$ в СМ должен приближать ее к нулю на масштабе Планка. 


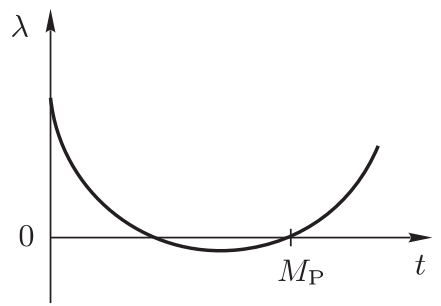

a

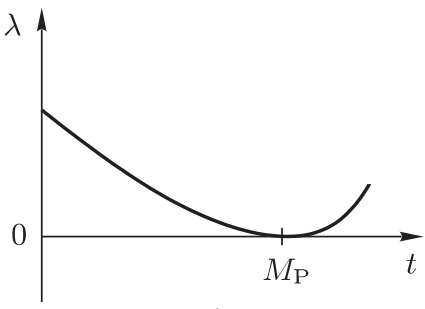

6

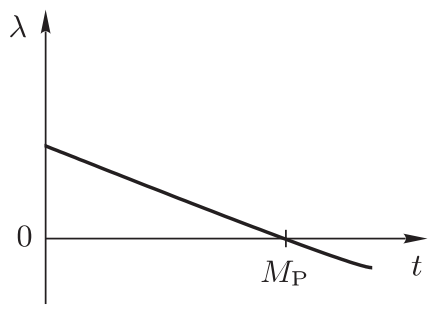

B

Рис. 2. Эволюция константы самодействия Хиггса $\lambda$ в СM с граничным условием $\lambda\left(M_{\mathrm{P}}\right)=0$.

Но это еще не все. Для непротиворечивости теории константа $\lambda(\mu)$ должна быть положительна при всех масштабах энергии. Найдем следствия такого требования, для чего рассмотрим эволюцию $\lambda$ согласно СМ при $\mu<M_{\mathrm{P}}$ с граничным условием (9). На рис. 2 показаны три различных возможности. Случай "а", когда $\lambda$ достигает нуля ниже масштаба Планка, исключается: при этом СМ разрушается ниже масштаба $M_{\mathrm{P}}$. Случай “в" потенциально опасен: отрицательное значение $\beta_{\lambda}^{\mathrm{SM}}$ при $k=M_{\mathrm{P}}$ в силу непрерывности приведет $\lambda$ к отрицательной величине над масштабом Планка. Другими словами, не только скалярное самодействие должно быть близко к нулю, но также и его бета-функция в СМ должна быть мала при $k=M_{\mathrm{P}}$ :

$$
\beta_{\lambda}^{\mathrm{SM}}\left(M_{\mathrm{P}}\right) \approx 0
$$

Насколько точно должны быть удовлетворены условия (9), (10), зависит от конкретных значений аномальных размерностей $a_{h}$ и требует численного решения уравнений РГ. Важно, что существуют два условия вместо одного, и это позволяет фиксировать (или по крайней мере ограничить) массы бозона Хиггса и топ-кварка одновременно.

Для большей точности при численных расчетах мы использовали двухпетлевые уравнения РГ. Низкоэнергетические константы связи были выражены через физические параметры в однопетлевом приближении (см. работы [44], [51], а также [43]). Ниже мы опишем наиболее существенные особенности наших результатов.

Требование положительности $\lambda$ при всех масштабах энергии приводит к жестким ограничениям массы топ-кварка. Ограничение снизу $m_{\mathrm{t}} \gtrsim 170$ ГэВ практически не зависит от аномальных размерностей $a_{h}$. По существу, если $m_{\mathrm{t}}<170$ ГэВ, то мы получим поведение, показанное на рис. 2a, приводящее к неустойчивому вакууму. Бо́льшие значения $m_{\mathrm{t}}$ соответствуют модели, изображенной на рис. 2 в. Если величины $a$ и $a_{y}$ достаточно велики, то постоянные $g_{i}$ и $y_{\mathrm{t}}$ быстро обращаются в нуль при $k>M_{\mathrm{P}}$, приводя к малой величине $\beta_{\lambda}^{\mathrm{SM}}$ выше масштаба Планка и, таким образом, к разумному поведению $\lambda$. Если значения $a$ и $a_{y}$ меньше, то абсолютное значение $\beta_{\lambda}^{\mathrm{SM}}$ увеличивается сразу же над масштабом Планка, толкая $\lambda$ в отрицательную область. Таким образом, верхний предел для массы топ-кварка, полученный из условия положительности $\lambda$, существенно зависит от $a$ и $a_{y}$. Например, для $a=a_{y}=-1$ и $a_{\lambda}=3$ допустимые траектории РГ существуют при самых разных массах топ-кварка: значение $m_{\mathrm{t}}=171.3$ ГэВ ведет к $m_{\mathrm{H}} \simeq 126$ ГэВ, тогда как 
$m_{\mathrm{t}}=230$ ГэВ требует $m_{\mathrm{H}} \simeq 227$ ГэВ. Выбор $a=a_{y}=-0.25$ и $a_{\lambda}=3$ ведет к ограничению сверху $m_{\mathrm{t}} \lesssim 174$ ГэВ, что очень близко к нижней границе. Тот факт, что экспериментальное значение массы топ-кварка удивительно близко к нижней границе (и к верхней границе для достаточно малого $a_{y}$ ), можно считать аргументом в пользу идей, выдвинутых в настоящей статье.

Теперь выберем экспериментальное значение массы топ-кварка и определим массу бозона Хиггса. Предсказание почти не чувствительно к конкретным значениям параметров $a, a_{y}$ и $a_{\lambda}$ и имеет вид

$$
m_{\mathrm{H}}=m_{\min },
$$

где $m_{\min }$ задана выражением (6). Нетрудно понять, почему это происходит. Поведение $\lambda$ в $\mathrm{CM}$, соответствующее $m_{\mathrm{H}}=m_{\min }$ и $m_{\mathrm{t}}=173.1$ ГэВ, в точности совпадает с тем, которое показано на рис. 2б. Уменьшение $m_{\mathrm{H}}$ приводит к поведению $\lambda(t)$, изображенному на рис. $2 \mathrm{a}$, что невозможно. Увеличение $m_{\mathrm{H}}$ приводит к положительной константе $\lambda\left(M_{\mathrm{P}}\right)$, причем при $a_{\lambda}>0$ функция $\lambda(M)$ стремится к бесконечности на масштабе выше масштаба Планка, что также невозможно. В последнем случае поведение можно изменить при условии, что взаимодействие Юкавы имеет негауссову фиксированную точку $a_{y}=a_{y}^{\text {crit }}$, и это приводит к существованию нетривиальной фиксированной точки для $\lambda$. Взяв в качестве примера $a=-1$ и $a_{y} \simeq-0.005$, получим $\lambda^{*}<0.043$. В результате предсказанная масса бозона Хиггса смещается вверх не более чем на 8 ГэВ. Взяв меньшие $a$, получим меньший сдвиг. Такая ситуации, однако, требует некоторой тонкой подгонки и поэтому выглядит маловероятной.

Наше предсказание (11) (или (5), если значение $a_{\lambda}$ в самом деле отрицательно) может быть проверено на БАК. Учитывая тот факт, что точность измерения массы бозона Хиггса на БАК может достигать 200 МэВ, весьма желательно уменьшить теоретическую неопределенность и экспериментальную погрешность в определении массы топ-кварка и константы сильного взаимодействия. Как обсуждалось в работе [43], теоретическую неопределенность можно уменьшить с 2.2 ГэВ до 0.4 ГэВ, если заменить однопетлевое сопоставление констант связи с физическими параметрами на электрослабых масштабах и двухпетлевой бег вплоть до масштаба Планка на двухпетлевое сопоставление и трехпетлевой бег. Отметим, что трехпетлевые бета-функции для СМ до сих пор не известны, а двухпетлевое сопоставление никогда не проводилось.

Предсказание $m_{\mathrm{H}} \approx m_{\text {min }}$ справедливо не только при предположении, что СМ в комбинации с гравитацией описывает всю физику, относящуюся к бегу констант связи. Оно распространяется и на многие обобщения СМ и гравитации, возможно, включая даже теории с дополнительными пространственными измерениями. Конечно, при рассмотрении большего класса моделей точность предсказания становится слабее. Тем не менее для справедливости предсказания $m_{\mathrm{H}} \approx m_{\min }$ необходимы только два ключевых условия. Во-первых, выше масштаба перехода $k_{\mathrm{tr}}$ РГ-эволюция должна быстро вести четверное скалярное взаимодействие к приближенной фиксированной точке $\lambda=0$, которая возмущена лишь малыми вкладами в $\beta_{\lambda}$ от взаимодействия Юкавы и калибровочного взаимодействия. Это обычно происходит при достаточно большой аномальной размерности $a_{\lambda}>0$. Во-вторых, вблизи $k_{\mathrm{tr}}$ должен иметь место переход к бегу, характерному для СМ в низкоэнергетическом режиме. Этот переход в действительности может приводить к некоторому 
расщеплению масштабов вследствие “пороговых эффектов", как в теориях великого объединения на масштабе вблизи $k_{\mathrm{tr}}$. Достаточно того, что эти эффекты не ведут к быстрому росту $\lambda$ в пороговой области. Это справедливо в том случае, когда не зависящие от $\lambda$ вклады в $\beta_{\lambda}$ включают в себя только достаточно слабые взаимодействия, которые учитываются как поправки в пороговой области, простирающейся всего на несколько порядков по величине.

Приведем некоторые комментарии.

1. Тот удивительный факт, что скалярное самодействие в СМ равно нулю вместе со своей бета-функцией на масштабе Планка для частных значений масс топ-кварка и бозона Хиггса, впервые (насколько нам известно) был отмечен в работе [52]. В этой работе в качестве гипотезы выдвинут “принцип многократной точки”, согласно которому эффективный потенциал для поля Хиггса должен иметь два минимума: один, соответствующий нашему вакууму, и другой, который должен существовать на масштабе Планка. Наша мотивация совершенно другая. Хотя смысл "принципа многократной точки" остается для нас неясным, мы хотели бы отметить, что вытекающее из него предсказание массы бозона Хиггса совпадает с нашим (конкретные числа в статье [52] другие, поскольку они основаны на однопетлевом расчете).

2. Найденные нами значения массы бозона Хиггса согласуются с возможностью инфляции, обусловленной бозоном Хиггса СМ [10]. Такая инфляция требует непротиворечивой $\mathrm{CM}$ вплоть до меньших чем $M_{\mathrm{P}}$ масштабов энергии, $k \sim M_{\mathrm{P}} / \xi$, где $\xi=700 \div 10^{5}$ - значение константы неминимального взаимодействия поля Хиггса со скалярной кривизной Риччи [43], [53] (см. также работы [54], [55]). Меньшие значения $\xi$ соответствуют меньшим массам бозона Хиггса.

3. В настоящей статье мы неявно предполагаем, что масштаб Ферми задан равным своему экспериментальному значению. В статьях [25], [27] найдено, что в системе со скалярным полем и гравитацией аномальная размерность скалярной массы отрицательна, поэтому константа взаимодействия существенна (и, таким образом, непредсказуема). Если для СМ это действительно так, то малость масштаба Ферми по сравнению с масштабом Планка остается загадкой. Наоборот, если аномальная размерность в рамках СМ оказывается положительной, то для непротиворечивости теории необходимо положить масштаб Ферми равным нулю в асимптотической области. Если последнее предположение окажется справедливым, то оно в конечном счете может прояснить причину огромной разницы между электрослабым масштабом и масштабом Планка.

\section{4. ЗАКЛЮЧЕНИЕ}

Мы обсудили возможность того, что СM, дополненная асимптотически безопасной гравитацией, может играть роль фундаментальной, а не просто эффективной теории поля. Мы показали, что это возможно в том случае, когда вклады от гравитации в бег констант взаимодействия Юкавы и Хиггса имеют подходящие знаки. Предсказана масса бозона Хиггса $m_{\mathrm{H}}=m_{\min } \simeq 130$ ГэВ с погрешностью несколько ГэВ для случая, когда все константы взаимодействия СМ за исключением константы самодействия Хиггса $\lambda$ асимптотически свободны, а $\lambda$ сильно притягивается к приближенной фиксированной точке $\lambda=0$ РГ-потоком в высокоэнергетической

5 Теоретическая и математическая физика, т. 170, № 2, 2012 г. 
области (в пределе исчезающих взаимодействия Юкавы и калибровочного взаимодействия). Этого можно достичь с помощью положительной аномальной размерности для бегущей константы $\lambda$, вызванной гравитацией. Похожее предсказание остается справедливым и для обобщения СМ до теории великого объединения при условии, что расщепление между масштабом объединения и масштабом Планка остается умеренным и все важные взаимодействия появляются в области перехода как малые возмущения. Обнаружение на БАК скаляра Хиггса с массой, приблизительно равной 130 ГэВ, было бы серьезным указанием на отсутствие новой физики, влияющей на бег констант связей в СМ между масштабами Ферми и Планка.

Благодарности. Работа частично поддержана Swiss National Science Foundation и Alexander von Humboldt Foundation.

\section{Список литературы}

[1] S. Weinberg, "Ultraviolet divergences in quantum theories of gravitation", General Relativity: An Einstein Centenary Survey, eds. S. W. Hawking, W. Israel, Cambridge Univ. Press, Cambridge, 1979, 790-831.

[2] M. Niedermaier, M. Reuter, Living Rev. Rel., 9 (2006), 2006-5, 173 pp.

[3] M. Shaposhnikov, Is there a new physics between electroweak and Planck scales?, arXiv: 0708.3550.

[4] A. Boyarsky, O. Ruchayskiy, M. Shaposhnikov, Ann. Rev. Nucl. Part. Sci., 59:1 (2009), 191-214, arXiv: 0901.0011.

[5] C. Wetterich, Nucl. Phys. B, 302:4 (1988), 668-696.

[6] C. Wetterich, Nucl. Phys. B, 302:4 (1988), 645-667.

[7] C. Wetterich, Phys. Rev. Lett., 90:23 (2003), 231302, 4 pp., arXiv: hep-th/0210156; 102:14 (2009), 141303, 4 pp., arXiv: 0806.0741; The cosmological constant and higher dimensional dilatation symmetry, arXiv: 0911.1063.

[8] M. E. Shaposhnikov, D. Zenhäusern, Phys. Lett. B, 671:1 (2009), 187-192, arXiv: hep-th/0809.3395.

[9] M. E. Shaposhnikov, D. Zenhäusern, Phys. Lett. B, 671:1 (2009), 162-166, arXiv: 0809.3406.

[10] F. L. Bezrukov, M. E. Shaposhnikov, Phys. Lett. B, 659:3 (2008), 703-705, arXiv: 0710.3755.

[11] S. Weinberg, Phys. Rev. D, 81:8 (2010), 083535, 8 pp., arXiv: 0911.3165.

[12] M.E. Shaposhnikov, C. Wetterich, Phys. Lett. B, 683:2-3 (2010), 196-200, arXiv: 0912.0208.

[13] A. M. Polyakov, Phys. Lett. B, 59:1 (1975), 79-81; E. Brézin, J. Zinn-Justin, Phys. Rev. Lett., 36:13 (1976), 691-694.

[14] R. Gastmans, R. Kallosh, C. Truffin, Nucl. Phys. B, 133:3 (1978), 417-434.

[15] S. M. Christensen, M. J. Duff, Phys. Lett. B, 79:3 (1978), 213-216.

[16] H. Kawai, M. Ninomiya, Nucl. Phys. B, 336:1 (1990), 115-145.

[17] K. G. Wilson, J. B. Kogut, Phys. Rept., 12:2 (1974), 75-199.

[18] J. Ambjørn, J. Jurkiewicz, R. Loll, Phys. Rev. Lett., 93:13 (2004), 131301, 4 pp., arXiv: hep-th/0404156.

[19] J. Ambjørn, J. Jurkiewicz, R. Loll, Quantum gravity as sum over spacetimes, arXiv: 0906.3947.

[20] J. Polchinski, Nucl. Phys. B, 231:2 (1984), 269-295.

[21] C. Wetterich, Phys. Lett. B, 301:1 (1993), 90-94.

[22] M. Reuter, Phys. Rev. D, 57:2 (1998), 971-985, arXiv: hep-th/9605030. 
[23] A. Codello, R. Percacci, C. Rahmede, Ann. Physics, 324:2 (2009), 414-469, arXiv: 0805.2909.

[24] M. R. Niedermaier, Phys. Rev. Lett., 103:10 (2009), 101303, 4 pp.

[25] R. Percacci, D. Perini, Phys. Rev. D, 68:4 (2003), 044018, 19 pp., arXiv: hep-th/0304222.

[26] J. E. Daum, U. Harst, M. Reuter, Running gauge coupling in asymptotically safe quantum gravity, arXiv: 0910.4938.

[27] G. Narain, R. Percacci, Renormalization group flow in scalar-tensor theories. I, arXiv: 0911.0386.

[28] S.P. Robinson, F. Wilczek, Phys. Rev. Lett., 96:23 (2006), 231601, 4 pp., arXiv: hep-th/0509050.

[29] A. R. Pietrykowski, Phys. Rev. Lett., 98:6 (2007), 061801, 4 pp., arXiv: hep-th/0606208.

[30] D. J. Toms, Phys. Rev. D, 76:4 (2007), 045015, 5 pp., arXiv: 0708.2990.

[31] D. J. Toms, Phys. Rev. Lett., 101:13 (2008), 131301, 4 pp., arXiv: 0809.3897.

[32] O. Zanusso, L. Zambelli, G. P. Vacca, R. Percacci, Phys. Lett. B, 689:2-3 (2010), 90-94, arXiv: 0904.0938.

[33] D. Ebert, J. Plefka, A. Rodigast, Phys. Lett. B, 660:5 (2008), 579-582, arXiv: 0710.1002.

[34] K. Nakemura et al. (Particle Data Group), J. Phys. G, 37:7A (2010), 075021, 1422 pp.

[35] I. L. Shapiro, Class. Quantum Grav., 6:8 (1989), 1197-1201.

[36] L. Maiani, G. Parisi, R. Petronzio, Nucl. Phys. B, 136:1 (1978), 115-124.

[37] N. Cabibbo, L. Maiani, G. Parisi, R. Petronzio, Nucl. Phys. B, 158:2-3 (1979), 295-305.

[38] M. Lüscher, P. Weisz, Nucl. Phys. B, 290:1 (1987), 25-60; T. Papenbrock, C. Wetterich, Z. Phys. C, 65:3 (1995), 519-535, arXiv: hep-th/9403164.

[39] M. Lindner, Z. Phys. C, 31:2 (1986), 295-300.

[40] Н. В. Красников, ЯФ, 28 (1978), 549-551.

[41] P. Q. Hung, Phys. Rev. Lett., 42:14 (1979), 873-876.

[42] H. D. Politzer, S. Wolfram, Phys. Lett. B, 82:2 (1979), 242-246; Erratum 83:3-4 (1979), $421-422$.

[43] F. Bezrukov, M. E. Shaposhnikov, JHEP, 07 (2009), 089, 24 pp., arXiv: 0904.1537.

[44] J. R. Espinosa, G. F. Giudice, A. Riotto, JCAP, 05 (2008), 002, 24 pp., arXiv: 0710.2484.

[45] J. Ellis, J. R. Espinosa, G. F. Giudice, A. Hoecker, A. Riotto, Phys. Lett. B, 679:4 (2009), 369-375, arXiv: 0906.0954.

[46] G. Altarelli, G. Isidori, Phys. Lett. B, 337:1-2 (1994), 141-144.

[47] J. A. Casas, J. R. Espinosa, M. Quirós, Phys. Lett. B, 342:1-4 (1995), 171-179, arXiv: hep-ph/9409458.

[48] J. A. Casas, J.R. Espinosa, M. Quirós, Phys. Lett. B, 382:4 (1996), 374-382, arXiv: hep-ph/9603227.

[49] T. Hambye, K. Riesselmann, Phys. Rev. D, 55:11 (1997), 7255-7262, arXiv: hep-ph/9610272.

[50] L. Griguolo, R. Percacci, Phys. Rev. D, 52:10 (1995), 5787-5796, arXiv: hep-th/9504092.

[51] M. X. Luo, Y. Xiao, Phys. Rev. Lett., 90:1 (2003), 011601, 4 pp., arXiv: hep-ph/0207271.

[52] C. D. Froggatt, H. B. Nielsen, Phys. Lett. B, 368:1-2 (1996), 96-102, arXiv: hep-ph/9511371.

[53] F. L. Bezrukov, A. Magnin, M. Shaposhnikov, Phys. Lett. B, 675:1 (2009), 88-92, arXiv: 0812.4950 .

[54] A. De Simone, M. P. Hertzberg, F. Wilczek, Phys. Lett. B, 678:1 (2009), 1-8, arXiv: 0812.4946 .

[55] A. O. Barvinsky, A. Yu. Kamenshchik, C. Kiefer, A. A. Starobinsky, C. Steinwachs, JCAP, 12 (2009), 003, arXiv: 0904.1698. 